International Mathematical Forum, 2, 2007, no. 21, 1025 - 1032

\title{
On Weak Central Extensions and Perfect Topological Groups
}

\author{
H. Sahleh \\ Department of Mathematics \\ Guilan University, P.O. Box 1914, Rasht, Iran \\ sahleh@guilan.ac.ir
}

\begin{abstract}
Let $G$ be a topologically perfect group (i.e. $G=\overline{[G, G]}$ ) and $M(G)$ the topological Schur multiplier of $G$. In this paper we show that $G$ has a weak universal central extension whose center is the Schur multiplier.
\end{abstract}

\section{Mathematics Subject Classification: 22A05}

Keywords: Free topological group; Schur multiplier; universal extension

\section{Introduction}

Perfect groups are important object of study in group theory. For a topologist, perfect groups tend to be those that arise in certain interesting situations. For example via the study of fundamental groups. For any discrete group $G$ ,$H_{1}(G)=G_{a b}$. For any topological space $X, H_{1}(X)=\pi_{1}(X)_{a b}$. The case where $X$ is the classifying space of $G$, the Eilenberg-Maclane space $K(G, 1)$, reduces to

$$
H_{1}(G)=H_{1}(K(G, 1))=\pi_{1}(K(G, 1))_{a b}=G_{a b}
$$

Hence for classes of space $X$ for which homology groups are easily calculated, perfect subgroups of the fundamental group have a role to play in calculation of $\pi_{1}(X)$. For example if $H_{1}(X)=0$, then $\pi_{1}(X)$ is perfect.

A good invariant of any group $G$ is its abelianization $G_{a b}$. The $G_{a b}$ is characterized by the universal property that every continuous group homomorphism factors uniquely through $G \rightarrow G_{a b}$. The abelianizations are indeed 
easer object to deal with than arbitrary groups. One way to get more information about a group is the case where $G$ has trivial abelinization. In general case ,the information lost in abelianization is measured by the kernel of $G \rightarrow G_{a b}$ (for more on perfect groups see [2]). In this paper we consider topologically perfect groups and will show that such a group has a universal topological extension whose kernel is the Schur multiplier.

All spaces are assumed to be Tychanov (completely regular, Hausdorff).A topological extension of $G$ by $N$, denoted by $(G, \pi)$, is a short exact sequence $0 \rightarrow N \stackrel{i}{\rightarrow} Q \stackrel{\pi}{\rightarrow} G \rightarrow 0$, where $i$ is a topological embedding onto a closed subgroup,$\phi$ a topological isomorphism of $G / N$ onto $G$. The extension is central if $N$ is in the center of $G$. We consider extensions with a continuous section i.e. $u: G \rightarrow Q$ such that $\pi u=I d$. For example, if $G$ is a connected locally compact group ,then any topological extension of $G$ by a connected simply connected Lie group has a continuous section [S,theorem 2].

\section{Perfect groups and the Schur multiplier}

In this section we consider the Schur multiplier in the topological context. We recall an example which shows that a perfect topological group need not be perfect.

The free topological group is in Markov sense[5]: If $X$ is completely regular ,then the (Markov) free topological group on $X$ is the group $F(X)$ equipped with the finest group topology inducing the given topology on $X$ as a subspace. Such a topology always axists [5], and has the universal property of the following kind:every continuous mapping $f$ from $X$ to an arbitrary topological group $G$ lifts to a unique continuous homomorphism

$$
g: F(X) \rightarrow G
$$

i.e.the restriction of $g$ to $X$ is $f$. For information on free topological groups see $[5,4]$.

Let $G$ be a topological group . A free topological presentation of $G$ is a short exact sequence $0 \rightarrow R \rightarrow F \stackrel{\pi}{\rightarrow} G \rightarrow 0$ with a continuous section $u: G \rightarrow F$ such that $\pi u=I d_{G}$ where $F$ is a free topological group in Morkov[6] sense. Consider the abelian group $R \cap[F, F] / \overline{[R, F]}$. We call this group the Schur multiplier of $G$ denoted by

$$
M(G)=R \cap[F, F] / \overline{[R, F]}
$$

By Alperin et al[1], $M(G)$ is independent of the choice of the presentation. 
The following example shows that a topologically perfect group need not be perfect.

Recall that a topological group $G$ is perfect if $[G, G]$ is dense in $G$.

Example [3]. Let $\widetilde{S L_{2}(R)}$ be the universal covering of $S L_{2} R$, special real linear group. Let $\alpha \in S L_{2}(R)$ be central element of infinite order. Let $\alpha$ be irrational rotation in the circle group $T$. Consider the group

$$
G=\widetilde{S L_{2}(R)} /<\alpha, \alpha>
$$

then $G$ is topologically perfect[see 3].

\section{Central extensions and perfect groups}

If $G$ is a perfect group, then there exists a universal extension $e=(U, \pi)$ of $G$ which has the property that for any other extension $e^{\prime}=\left(G_{1}, \pi^{\prime}\right)$ there exists a unique homomorphism $\phi: U \rightarrow G_{1}$ with $\pi^{\prime} \phi=\pi$. The kernel of $\pi$ is the second homology group of $G([8],[6])$. In this section we consider the idea in a topological context and will show that for a topological perfect group i.e. $G=\overline{[G, G]}$ the weak universal extension exists and its kernel is the Schur multiplier.

Definition 2.1. A weak central extension of $N$ by $Q$ is an exact sequence $0 \rightarrow N \rightarrow G \rightarrow Q \rightarrow 0$ with $N$ a central subgroup and $\pi$ an onto continuous homomorphism( not necessarily open). A (weak) central extension of $N$ by $Q$ is (weak) universal if for any other (weak) central extension , $0 \rightarrow N^{\prime} \rightarrow G^{\prime} \stackrel{\pi^{\prime}}{\rightarrow}$ $Q \rightarrow 0$ there is one and only one continuous homomorphism from $G$ to $G^{\prime}$ over $Q$ such that the following diagram commutes:

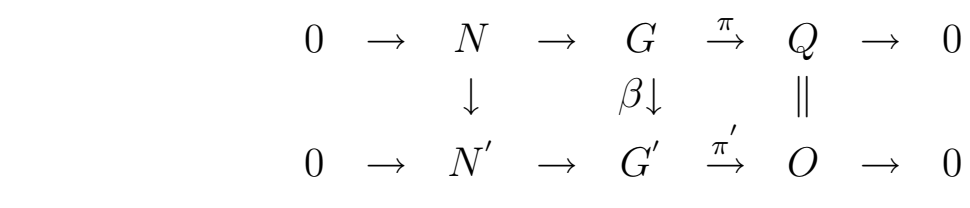

$\pi^{\prime} \beta=\pi$

Lemma 2.2 Let $e=(G, \pi)$ and $e^{\prime}=\left(G^{\prime}, \pi^{\prime}\right)$ be two central extensions of $Q$. If $G$ is topologically perfect i.e. $G=\overline{[G, G]}$, then there is at most on continuous homomorphismfrom $G^{\prime}$ over $Q$.

Proof. Let $f_{1}, f_{2}$ be two continuous homomorphisms from $G$ to $G^{\prime}$ over $Q$ i.e. $\pi f_{1}=\pi, \pi^{\prime} f_{2}=\pi$. Let $y, z \in G$. Now $\pi^{\prime} f_{1}(y)=\pi$ and $\pi^{\prime} f_{2}(y)=\pi$. Hence 
, there are $c, c^{\prime}$ in the kernel of $Q$ such that $f_{1}(y)=f_{2}(y) c, f_{1}(z)=f_{2}(z) c^{\prime}$. Since $f_{1}$ and $f_{2}$ are homomorphisms and $c, c^{\prime}$ are in the center of $G^{\prime}$ we have,

$$
\begin{aligned}
f_{1}[y, z] & =f_{1}\left[y z y^{-1} z^{-1}\right] \\
& =f_{1}(y) f_{1}(z) f_{1}\left(y^{-1}\right) f_{1}\left(z^{-1}\right) \\
& =f_{2}(y) c f_{2}(z) c^{\prime} c^{-1} f_{2}(y)^{-1} c^{\prime-1} f_{2}(z)^{-1} \\
& =f_{2}(y) f_{2}(z) f_{2}\left(y^{-1}\right) f_{2}\left(z^{-1}\right) \\
& =f_{2}\left(y z y^{-1} z^{-1}\right)=f_{2}[y, z]
\end{aligned}
$$

Hence $f_{1}, f_{2}$ agree on the commutator subgroup of $G$. Since $[G, G]$ is dense in $G$ so $f_{1}, f_{2}$ are the same on $G$.

Lemma 2.3 Let $e=(G, \pi)$ be a central extension of $Q$. If $G$ is not topologically perfect then for a suitably chosen $e^{\prime}=\left(G^{\prime}, \pi^{\prime}\right)$ a central extension of $Q$, there exists more than one continuous homomorphism from $G$ to $G^{\prime}$ over $Q$.

Proof. If $G$ is not topologically perfect then there is a non zero continuous homomorphism from $G$ to some abelian topological group (for example $\phi$ : $G \rightarrow G / \overline{[G, G]})$. Let $e^{\prime}: 0 \rightarrow N^{\prime} \rightarrow G^{\prime} \rightarrow Q \rightarrow 0$ be the split extension of $N^{\prime}$ by $Q$ with $\pi^{\prime}\left(q, n^{\prime}\right)=q, q \in Q, n^{\prime} \in N^{\prime}$. Clearly $e$ is central extension. Setting $f_{1}(g)=(\pi(g), 1)$ and $f_{2}(g)=(\pi(g), \phi(g))$, we obtain two distinct continuous homomorphisms from $G$ to $G^{\prime}$ over $Q$ because for any $g \in G$,

$$
\begin{gathered}
\pi^{\prime} f_{1}(g)=\pi^{\prime}((\pi(g), 1)=\pi(g) \\
\pi^{\prime} f_{2}(g)=\pi^{\prime}(\pi(g), \phi(g))=\pi(g)
\end{gathered}
$$

Since $\pi$ and $\phi$ are continuous then $f_{1}, f_{2}$ are continuous. These two maps are distinct because $\phi$ is a non zero homomorphism

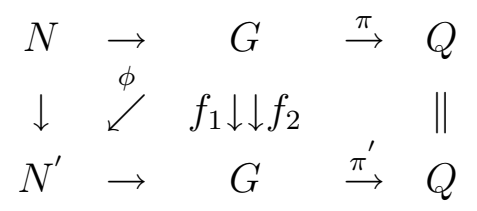

Lemma 2.4 If $e=(G, \pi)$ is a central extension of a topologically perfect group $Q$ i.e. $Q=\overline{[Q, Q]}$, then $G=\overline{[G, G]}$ is topologically perfect and maps onto Q.In particular, $\left(G^{\prime},\left.\pi\right|_{G^{\prime}}\right)$ is a weak central extension.

Proof. Let $e=(G, \pi)$ be a central extension of $Q$. since $\pi$ is a homomorphism ,it maps $[G, G]$ onto $[Q, Q]$. Let $z \in G, \pi(z)=y$ and $V$ be a 
neighborhood of $z$. So $y \in \overline{[Q, Q]}$. Hence there is a sequence $\left(y_{n}\right)$ in $[Q, Q]$ such that $y_{n}$ converges to $y$. Sinec $\pi$ is open,$\pi(V)$ is an open set containing $y$ . So $\pi(V) \cap[Q, Q] \neq \emptyset$.

Therefore, $V \cap[G, G] \neq \emptyset$. It follows that $z$ is a limit point of $[G, G]$ i.e. $z \in \overline{[G, G]}$. Thus every $g \in G$ can be written as a product $x^{\prime} c$ with $x^{\prime} \in \overline{[G, G]}$ and $c \in K e r \pi$. Therefore, if $g_{1}, g_{2} \in G$, then $g_{1}=x_{1}^{\prime} c_{1}, g_{2}=x_{2}^{\prime} c_{2}$

$$
\left[g_{1}, g_{2}\right]=\left[x_{1}^{\prime} c_{1}, x_{2}^{\prime} c_{2}\right]=\left[x_{1}^{\prime}, x_{2}^{\prime}\right]
$$

i.e. $[G, G]=\left[G^{\prime}, G^{\prime}\right]$. Hence $G^{\prime}=\overline{[G, G]}=\overline{\left[G^{\prime}, G^{\prime}\right]}$.

Now we define the backward induced extension in the category of topological groups.

Let $\nu: Q_{1} \rightarrow Q$ and $\pi: G \rightarrow Q$ be continuous . The backward extension of $\{\nu, \pi\}$ is a group $G^{(2)}$ and two continuous homomorphisms $\pi_{0}: G^{(2)} \rightarrow Q$ and $\beta: G \rightarrow Q$ such that $\pi_{0} \nu=\beta \pi$ and with the property that given any topological group $\Gamma$ and $\alpha: \Gamma \rightarrow G^{(2)}, \delta: \Gamma \rightarrow G$ such that $\nu \alpha=\pi \delta$ then there exists a unique continuous homomorphism $\gamma: \Gamma \rightarrow G^{(2)}$ so that $\delta=\beta \gamma$ and $\alpha=\pi_{0} \gamma$. By $[\mathrm{C}]$ backward extension exists. Note that $G^{(2)}=\{(g, u) ; \nu(u)=$ $\pi(g)\}$ and it is a subgroup of $G \times Q_{1}$ together with the restriction of cononical projection from $G \times Q_{1}$ onto $G$ and $Q_{1}$ respectively. Remark. If the topological extension $e=(G, \pi)$ of $Q$ has a continuous section $u: Q \rightarrow G$ i.e. $\pi u=I d$, then $\sigma: Q \rightarrow G^{(2)}$ defined by $\sigma(q)=(u \nu(q), q)$ is a continuous section of $G^{(2)}$.

Lemma 2.5 A central extension $(U, \nu)$ of $Q$ is universal if and only if $U$ is topologically perfect and every central extension of $U$ splits.

Proof. Suppose that every central topological extension of $U$ splits and $U$ is topologically perfect. Let $e: N \rightarrow G \rightarrow Q$ be a central exrension of $N$ by $G$.Now we form the backward extension of $e$ as follows:

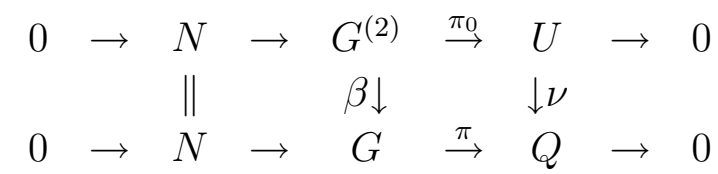

$\nu \pi_{0}=\pi \beta$. By[4,lemma 3.3], $\left(^{*}\right)$ is computative with the topological exact rows. The top row of $(*)$ is central since $N$ is a $U$-module via $\pi_{0}$ and a trivial $Q$-module. Hence there is a continuous homomorphism $s: U \rightarrow G^{(2)}$ with $\pi s=1_{U}$. Now $(\pi(\beta s))(u)=\nu\left(\pi_{0} s(u)\right)=\nu(u), u \in U$ and $\beta s$ is a continuous homomorphism. By lemma $2.2, \beta s$ is unique. Therefore $(U, \nu)$ is the universal 
central extension of $Q$.

Now let $(U, \nu)$ be the universal central extension of $Q$. By lemma 2.3, $U$ is topologically perfect. We show that every central extension of $U$ splits. Given a central extension $(G, \pi)$ of $U$, we will show that $(G, \nu \pi)$ is a central extension of $Q$. For if $\nu\left(\pi\left(g_{0}\right)\right)=1$, then $\pi\left(g_{0}\right)$ is in the center of $U$. Hence, the the map $f: G \rightarrow G, f(g)=g_{0} g g_{0}^{-1}, g \in G$ is a continuous homomorphism because

$$
\pi(f(g))=\pi\left(g_{0} g g_{0}^{-1}\right)=\pi\left(g_{0}\right) \pi(g) \pi\left(g_{0}^{-1}\right)=\pi(g), g \in G
$$

Restricting to $G^{\prime}=\overline{[G, G]}$ by lemmas 2.4 and 2.2 the resulting homomorphism from $G^{\prime}$ to $G$ over $U$ is the identity. Thus $g_{0}$ commutes with elements of $G^{\prime}$. But every $g \in G$ can be expressed as $g=g^{\prime} c, g^{\prime} \in G^{\prime}, c \in k e r f$ For if $g \in G$, since $\pi$ maps $G^{\prime}=\overline{[G, G]}$ onto $U$ there exists $g^{\prime} \in G^{\prime}$ with $\pi(g)=\pi\left(g^{\prime}\right)$. Hence $g=g^{\prime} c$ for $c \in k e r \pi$. So $g_{0}$ commutes with all elements of $G$. Since $\pi, \nu$ are open onto continuous homomorphisms so is $\nu \pi$. Thus $(G, \nu \pi)$ is a central extension of $Q$.Since $(U, \pi)$ is universal, there is a continuous homomorphism $S: U \rightarrow G$ over $Q$. The map $\pi S$ is a continuous map from $U$ to $U$ over $Q$; hence equals to the identity. Thus $S$ is a splitting. Therefore, every central extension of $U$ splits.

The next theorem gives the sufficient and necessary condition for a topological group to have the weak universal extension.

Theorem 2.6 A topological group $Q$ admits the weak universal central extension if and only if $Q$ is topologically perfect.

Proof. Let $(U, \nu)$ be the weak universal central extension of $Q$. By lemma $2.3, U$ is topologically perfect. By the continuity of $\nu$

$$
\nu \overline{[U, U]} \subset \overline{\nu[U, U]}=\overline{[Q, Q]}
$$

On the other hand $\nu \overline{[U, U]}=Q$. Hence $Q=\overline{[Q, Q]}$.

Conversely, if $Q$ is perfect choose a free topological presentation of $Q$,

$$
e: 0 \rightarrow R \rightarrow F \rightarrow Q \rightarrow 0
$$

and consider

$$
0 \rightarrow R / \overline{[R, F]} \rightarrow F / \overline{[R, F]} \rightarrow Q \rightarrow 0
$$

the centralizer of $(e)$. By lemma 2.4, $\overline{[F / \overline{[R, F]}, F / \overline{[R, F]}]}=\overline{[F, F] / \overline{[R, F]}} \rightarrow Q$ is a weak central extension . By $[6],[F, F]$ is closed in $F$, so $[F, F] / \overline{[R, F]}$ is closed. Hence

$$
[F, F] / \overline{[R, F]}=\overline{[F, F] / \overline{[R, F]}}
$$


We claim that $[F, F] / \overline{[R, F]} \rightarrow Q$ is the weak universal central extension of $Q$. Given any weak central extension $(G, \pi)$ of $Q$ with a continuous section $S$ : $Q \rightarrow G$, since $F$ is a free topological group there is a continuous homomorphism $\phi: F \rightarrow G$ over $Q$. Also $\phi[R, F]=1$ since $(G, \pi)$ is central. Now by the continuity of $\phi, \phi \overline{[R, F]}=1$. Thus $\phi$ induces a continuous homomorphism from $F / \overline{[R, F]}$ to $G$ over $Q$. Restricting this map to $[F, F] / \overline{[R, F]}$, we obtain the required homomorphism from $[F, F] / \overline{[R, F]}$ to $G$ over $Q$ which is unique by lemma 2.2 .

Proposition 2.7 Let $Q$ be a perfect topological group and the weak universal central extension $(U, \pi)$ of $Q$ exists and has a continuous section. Then the kernel of $\pi$ is isomorphic to $M(Q)$, the Schur multiplier of $Q$.

Proof. Let $N \rightarrow U \stackrel{\pi}{\rightarrow} Q$ be the universal central extension of $Q$ with a section $S: Q \rightarrow U, \pi S=I d$. By lemma 2.5, $U$ is perfect and every central extension of $U$ splits. By [1,theorem 2.3] $\operatorname{Hom}_{C}(M(U), M(U))=0$. This set contains the identity map, hence $M(U)=0$. By [1, proposition 1.8] there is an exact sequence

$$
M(U) \rightarrow M(Q) \rightarrow N \rightarrow U_{a b} \rightarrow Q_{a b} \rightarrow 0
$$

But $M(U)=0$ and, $U_{a b}=0, Q_{a b}=0$ because they are perfect. Therefore, $M(Q)$ is isomorphic to $N$.

\section{References}

[1] R.C. Alperin and H. Sahleh, Hopf,s Formula and the Schur multiplicator for topological groups, Kyungpook Math. Journal, 31 (1) (1991), 35-71.

[2] A.J. Berrick, A topologist,s view of perfect groups and acyclic groups, in :Invitations to Geometry and Topology ,in:Oxf.Texts Math.,vol. 7 (2002) ,Oxford University Press, Oxford, 1-28.

[3] E. Breuilard and T. Gelander, On dense free subgroups of Lie groups, J. Alg., 261 (2)(2003), 48-462.

[4] J.M. Castillo, On the "three-space" problem for locally quasi-covex topological groups , Arch. Math., 74 (2000), 253-262.

[5] E. Hewitt and K.A. Ross, Abstract Harmonic Analysis I, Academic Press, New York, 1963. 
[6] A.A. Markov, On free topological groups, Amer. Math. Soc. Transl., 30(1950), 11-88.

[7] C. Roger, Extensions centrales d'algebres de groupes de Lie de dimension infinite,algebres de Virasoro et generalisation, Rep. Math. Phys. 35 (1995), 225-266.

[8] A. Shtern, Remarks on Pseudocharacters and the Real Continuous Bounded Cohomology of Connected Locally Compact Groups, Annals of Global analysis and Geometry, 20 (2001), 199-221.

[9] C.A. Weibel, An introduction to homological algebra, Cambridge Stud. Adv. Math., 38 Cambridge Univ.Press, 1995 .

\section{Received: August 7, 2006}

